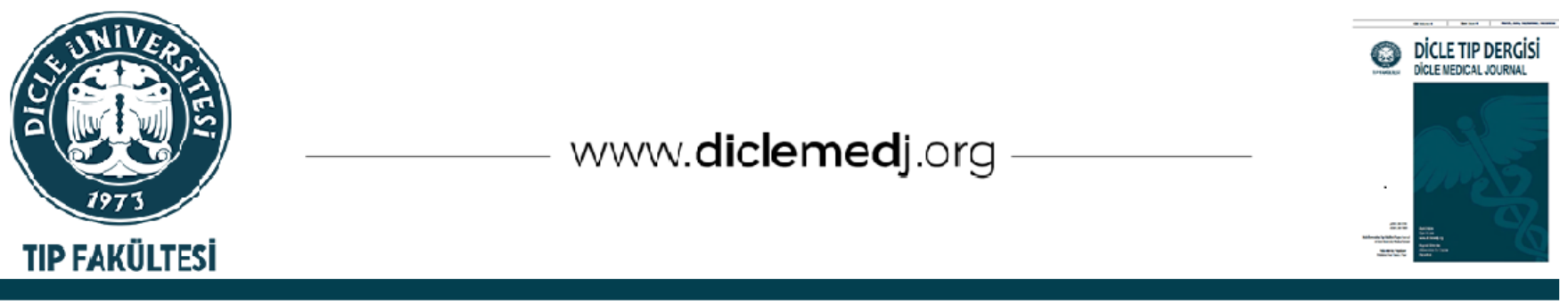

Original Article / Özgün Araştırma

\title{
The Determinants of Academic Achievement in Medical Students: a Cross-Sectional Designed Multifactorial Approach
}

\author{
Aslı Karakılıç ${ }^{D_{1}}$, Hayrettin Kara ${ }^{2,3}$, Haydar Ali Erken ${ }^{D_{1}}$, Gülten Erken ${ }^{D_{1}}$, Ahmet Burak Filik ${ }_{4}$ \\ 1 Department of Physiology, School of Medicine, Balıkesir University, Balıkesir, Turkey \\ 2 Nutritionist in Balıkesir University Research Hospital, Balıkesir, Turkey \\ 3 Department of Biochemistry, Faculty of Veterinary Medicine, Uludag University, Bursa, Turkey \\ 4 Medical student in Balıkesir University, School of Medicine, Balıkesir, Turkey
}

Received: 02.08.2021; Revised: 08.11.2021; Accepted: 22.11.2021

\begin{abstract}
Objective: Medical students have a heavy academic burden, hence, various factors can directly and easily affect their academic performance. In this study, we aimed to investigate the effects of hemoglobin levels, body composition, nutritional status and physical activity on academic achievement in medical students.

Methods: 121 students (69 males, 52 females) participated in the study. Body compositions were recorded; data concerning nutritional habits, food consumption frequency and physical activity was obtained using a survey. The hemoglobin and hematocrit values measured in the physiology practice were recorded and the scores obtained from the committee exam were considered as academic achievement criteria.
\end{abstract}

Results: Female students were found to be more successful than males $(p=0.012)$. Smokers $(p=0.007)$ and sugarybeverage consumers $(p=0.022)$ were also more unsuccessful. Males who did not consume coffee daily were more successful than those who did $(\mathrm{p}=0.015)$. Additionally, males with moderate physical activity levels were more successful than those who had low physical activity levels $(\mathrm{p}=0.04)$. Furthermore, there was a moderate positive correlation between academic achievement and sedentary behavior $(\mathrm{p}<0.05, \mathrm{r}=0.265)$.

Discussion: Based on our findings, we determined that smoking, coffee and sugary-beverage consumption may negatively affect academic success in medical students. Studying theoretical lessons daily for a sufficient period and getting moderate physical activity in leisure time can be recommended as tools for medical students to increase academic achievement.

Keywords: Academic achievement, body composition, physical activity, nutrition, medical education

DOI: 10.5798/dicletip.1037575

Correspondence / Yazışma Adresi: Aslı Karakılıç, Balıkesir University Faculty of Medicine Physiology Dept. Çă̆ış/Balıkesir10145/Turkey e-mail: asli.karakilic@balikesir.edu.tr 


\section{Tıp Öğrencilerinde Akademik Başarının Belirleyicileri: Kesitsel Tasarlanmış Multifaktöriyel Bir Yaklaşım}

Öz

Giriş: Tıp öğrencilerinin akademik yükünün fazla olması nedeniyle, çeşitli faktörler akademik başarılarını doğrudan ve kolayca etkileyebilmektedir. Bu çalışmada, tıp öğrencilerinde hemoglobin düzeyi, vücut kompozisyonu, beslenme durumu ve fiziksel aktivitenin akademik başarıya etkisini araştırmayı amaçladık.

Yöntemler: Çalışmaya 121 öğrenci (69 erkek, 52 kı) katılmıştır. Öğrencilerin vücut kompozisyonları kaydedilmiştir. Beslenme alışkanlıkları, besin tüketim sıklığı ve fiziksel aktivite verileri anket yöntemi ile elde edilmiştir. Fizyoloji uygulaması sırasında ölçülen hemoglobin ve hematokrit değerleri kayıt altına alınmış ve akademik başarı kriteri olarak öğrencilerin kurul sınavından aldıkları puanlar kullanılmıştır.

Bulgular: Kız öğrenciler erkeklere göre daha başarılı bulunmuştur $(p=0,012)$. Sigara içenler $(p=0,007)$ ve şekerli içecek tüketenler $(p=0,022)$ daha başarısız iken günlük kahve tüketmeyen erkekler tüketenlere göre daha başarılı olarak saptanmıştır $(\mathrm{p}=0,015)$. Ayrıca orta düzeyde fiziksel aktiviteye sahip erkekler, düşük fiziksel aktivite düzeyine sahip olanlardan daha başarılı sınav performansı sergilemiştir $(p=0,04)$. Ek olarak, akademik başarı ile sedanter davranış arasında orta düzeyde pozitif bir ilişki bulunmuştur $(\mathrm{p}<0,05, \mathrm{r}=0,265)$.

Tartışma: Bulgularımıza göre tıp öğrencilerinde sigara, kahve ve şekerli içecek tüketimi akademik başarıyı olumsuz etkilemektedir. Tıp öğrencilerinin akademik başarılarını artırmak için her gün teorik derslere yeterince zaman ayırmaları ve boş zamanlarında orta derecede fiziksel aktivite yapmaları önerilebilir.

Anahtar kelimeler: Akademik başarı, vücut kompozisyonu, fiziksel aktivite, beslenme, tıp eğitimi.

\section{INTRODUCTION}

Academic achievement in medical education is very important not only for medical students but also for society in general. Since incorrect interventions or treatments due to a lack of knowledge of physicians can result in the cost of human life, a graduate of medical school must have sufficient medical knowledge to implement the right decisions in vital situations.

To date, many factors have been associated with academic achievement. However, these factors have generally been addressed individually, or the research concentrated on children and adolescents. Thus, extensive studies addressing many factors simultaneously may provide deeper insight into various factors affecting academic performance ${ }^{1}$.

The academic burden of medical students is higher than other undergraduates. Therefore, physical, psychological and social factors may easily affect their academic achievement. In the literature, many factors affecting the academic success of students have been examined. To the best of our knowledge, no research focusing on the academic success of medical students on many modifiable physiological factors within a single study have yet been conducted. Instead of studying social and psychological factors, our objective was to clarify the relationship between academic achievement and various physiological parameters which are "modifiable". In addition, we aimed to offer suggestions and appropriate guidance to medical faculty students in order to enable them to increase their academic performance. In this study, the effects of hemoglobin level, body composition, physical activity and nutritional habits on academic success were investigated in medical students.

\section{METHODS}

\section{Participants}

This study was conducted on students in the 2nd year of the Balıkesir University School of Medicine in the 2018-2019 academic year. The 2nd year students were chosen for two reasons: they have generally overcome the adaptation 
period to the physical and social environment in the first year and have the highest theoretical burden in the medical faculty. Consequently, the psychogenic factors have been eliminated as much as possible. Since there are certain situations which can cause changes in physiological parameters that have the potential to affect students' academic achievement, students with chronic diseases (diabetes, hypertension, hypothyroidism, etc.), those who used medications continuously (hormones, antidiabetics, anti-hypertensives, antidepressants, sleeping pills, iron supplement etc.) or followed a special diet (low calorie diet, ketogenic diet, etc.) or who were younger than 18 and older than 25 were excluded. For example, chronic diseases such as diabetes, hypothyroidism, and the continued use of drugs (hormones, antidepressants, etc.) can affect the metabolism and yield have a direct effect on body composition, physical activity and nutritional habits. Based on these exclusion criteria, 12 students in total were not included. A total of 121 second-year students, (69 males and 52 females) aged 19-25 were included in the study. Post hoc, two-tailed power analysis was calculated as $87 \%$ with 0.05 type-1 error probability. Participation was entirely voluntary and students' written consents were obtained. The study protocol was approved by the Clinical Research Ethics Committee of Balıkesir University Faculty of Medicine (Decision date: 30.01.2019, Number: 2019/24).

\section{Measurements}

This study was implemented between November 2018 and January 2019 and lasted for a total of 3 months. Hemoglobin values were determined in November, questionnaire data (nutritional habits and physical activity status) was collected and body composition measurements were completed in DecemberJanuary. Hemoglobin measurements and questionnaire applications were conducted by the Balıkesir University Physiology
Department, while body composition measurements were obtained in the Balıkesir University Hospital Diet Polyclinic.

\section{Hemoglobin and Hematocrit}

The students' hemoglobin and hematocrit measurements were performed using a hemoglobinometer device (Mission Hb, ACON Laboratories, Inc. San Diego, USA) and obtained during the hemoglobin practice of physiology in November of the 2018-2019 academic year. This device works with 1 drop of blood taken from the fingertip via a lancet, without the need for blood to be stored, and provides results within 5-10 seconds.

\section{Academic Achievement}

Academic achievement was evaluated based on the scores of the 2nd Committee exam in January of the 2018-2019 academic year. It is known that the life span of an erythrocyte is 120 days. Thus, the 2nd Board exam was preferred as it was the closest exam to the date of student practice of hemoglobin measurement. Hence, we aimed to obtain more reliable results by keeping the time between the exam dates and the hemoglobin measurements at minimum.

Body Composition and Body Mass Index (BMI)

Students' height and weight were recorded by the same researcher with a weighing stadiometer device (Nanbaskul Scale DRMOD.85, Istanbul, Turkey) before lunch and without shoes. BMI was calculated as the weight $(\mathrm{kg})$ divided by the square of height $(\mathrm{m})$ and separated into two categories as $\mathrm{BMI}<25$ and $\mathrm{BMI} \geq 25^{2}$. In addition, body compositions were determined with an analyzer (MC-780MA body composition analyzer, Tanita Cooperation, Tokyo, Japan) which performs based on the bioelectrical impedance method. Body fat ratio values of $8-15 \%$ in men and $15-22 \%$ in women were considered as normal ranges 3 .

The Determination of Physical Activity Level and Nutritional Status 
A questionnaire was developed to determine the physical activity levels and nutritional status of the last three months ${ }^{4,5}$. The four-part questionnaire included questions concerning general information, physical activity, eating habits and frequency of food consumption, respectively.

General Information: This section contained questions about age, gender, smoking and alcohol use, chronic disease, medication, vitamin supplement and following a diet.

Physical activity: The Turkish version of the International Physical Activity QuestionnaireShort Form (IPAQ-SF), of which the validity and reliability study had previously been performed, was applied to determine the physical activity status ${ }^{6}$. The test-retest reliability coefficient of the Turkish IPAQ short form was $r=0.69, p<0.001$. There are 7 questions in total in the IPAQ short form and it allows the calculation of metabolic equivalent (MET) by determining the students' weekly physical activity frequency, duration and intensity levels. As a result, the weekly physical activity amounts were determined quantitatively in addition to being evaluated in 3 categories as low, medium and high. The MET limit values used for categorization are as follows: (1) <600 MET-min/wk sedentary, (2) 600-3000 MET-min/wk moderate, (3) >3000 MET-min/wk high physical activity level.

Eating habits: In this section, we questioned the habits for daily main meals, water, tea, coffee, beverage consumption and adding sugar to drinks.

Food consumption frequency: In this section of the questionnaire, the frequencies of dairy products, meat products, vegetable, fruit, grains, oil and sugar consumptions were determined. In addition to the content of these groups, the recommended daily amount of portions for individuals aged 18-49 for each food group were determined according to the
Turkey Nutrition Guideline ${ }^{4}$. The foods under each group title were indicated along with portion amounts (in $\mathrm{g}$ or $\mathrm{mL}$ ) and their equivalents (spoon, bowl, palm, cup etc.). The food consumption frequency was evaluated in 9 categories: "each meal", "each day", "5-6 times a week", "3-4 times a week", "1-2 times a week", "once in 15 days", "once a month", "rarely" and "never". In addition, the participants were asked to add cross marks to indicate the number of portions they consumed to the appropriate consumption frequency box. During data evaluation, a coefficient value corresponding to each food consumption frequency was determined. As we aimed to evaluate the food consumption scores of the last 3 months, the coefficient values were 270 (90 days $\mathrm{x} 3$ ) for "each meal", 90 for "each day", 72 (13 weeks x5.5) for "5-6 times a week", 46 (13 weeks x3.5) for "3-4 times a week", 20 (13 weeks x1.5) for " 1-2 times a week", 6 (90 days/15=6) for "once in 15 days", 3 (90 days/30=3) for "once a month ", 1 for "rarely" and 0 for "never". These coefficients were multiplied by the number of portions that the participants marked on the food consumption frequency. Consequently, the nutritional score was obtained for each food group. In addition, according to the daily recommended portion quantity of each food group, a 3-month sufficient consumption score was calculated separately for males and females. As result of the comparison with these limit scores, scores obtained from the participants were categorized as sufficient and insufficient consumption. Since there was no recommended limit of daily consumption for these groups, fat and sugar consumption was not categorized.

\section{Statistical Analysis}

All analyses of data were conducted via SPSS v.21.0 software. The independent t-test was conducted to examine the differences in academic achievement between two groups. For the data consisting of three groups, the 
difference between groups was evaluated with one-way ANOVA and post-hoc Bonferroni. The relationship between continuous data and academic achievement was investigated using the Pearson correlation analysis. $\mathrm{p}<0.05$ was considered statistically significant.

\section{RESULTS}

\section{Hemoglobin results}

The hemoglobin and hematocrit values for both females and males are provided in Table1.

When anemia criteria determined as $12 \mathrm{gr} / \mathrm{dl}$ for females and $13 \mathrm{gr} / \mathrm{dl}$ for males were Table I: Descriptive results for continuous variables applied ${ }^{7}, 7$ females were evaluated as being anemic while no males met the anemia criteria. The hemoglobin and hematocrit values of male students were observed to be significantly higher than female students $(\mathrm{p}<0.0001)$. No significant correlation was detected between hemoglobin and hematocrit values and academic achievement (Table 4).

Physical Activity Results

The physical activity and sedentary behavior details of males and females are provided in Table1.

\begin{tabular}{|c|c|c|c|c|c|c|}
\hline \multirow{2}{*}{ Characteristic } & \multicolumn{3}{|c|}{ Range } & \multicolumn{3}{|c|}{ Mean?SD } \\
\hline & Male & Female & Total & Male & Female & Total \\
\hline Age (years) & $19-25$ & $19-25$ & $19-25$ & $20,7 \pm 1,57$ & $20,1 \pm 1,03$ & $20,44 \pm 1,39$ \\
\hline Weight (kg) & $57,3-99,7$ & $44,3-87,4$ & $44,3-97,9$ & $75,27 \pm 10,84$ & $61,25 \pm 9,25$ & $69,39 \pm 12,3$ \\
\hline Height $(\mathrm{cm})$ & $162-189$ & $154-177$ & $154-189$ & $175,66 \pm 5,92$ & $164,03 \pm 5,87$ & $170,78 \pm 8,23$ \\
\hline BMI $\left(\mathrm{kg} / \mathrm{m}^{2}\right)$ & $18,93-36,18$ & $16,88-30,97$ & $16,88-36,18$ & $24,36 \pm 3,17$ & $22,79 \pm 3,37$ & $23,7 \pm 3,33$ \\
\hline Skeletal muscle mass (kg) & $44,5-73,4$ & $34,7-50,6$ & $34,7-73,4$ & $57,5 \pm 6,24$ & $41,9 \pm 3,57$ & $50,95 \pm 9,36$ \\
\hline Skeletal muscle ratio (\%) & $68,3-86,8$ & $57,3-80,7$ & $57,3-86,8$ & $76,93 \pm 4,46$ & $69,17 \pm 5,96$ & $73,67 \pm 6,4$ \\
\hline Body fat mass (kg) & $5-27,5$ & $7,7-34,6$ & $5-34,6$ & $14,69 \pm 5,35$ & $17,06 \pm 6,57$ & $15,69 \pm 5,97$ \\
\hline Body fat ratio (\%) & $8,6-28,1$ & $15,1-39,6$ & $8,6-39,6$ & $19,02 \pm 4,72$ & $27,14 \pm 6,27$ & $22,43 \pm 6,73$ \\
\hline MET-min/week & $248-5466$ & $198-4026$ & $198-5466$ & $2029,98 \pm 1521,52$ & $1525,08 \pm 960,66$ & $1820,08 \pm 1334,85$ \\
\hline $\begin{array}{l}\text { Sedentary behavior } \\
\text { (min/day) }\end{array}$ & $60-1080$ & $180-900$ & $60-1080$ & $468,27 \pm 214,62$ & $545,68 \pm 182,19$ & $500,45 \pm 204,32$ \\
\hline Hemoglobin (g/dl) & $13,1-17,6$ & $9,3-16,5$ & $9,3-17,6$ & $15,25 \pm 0,97$ & $13,2 \pm 1,3$ & $14,37 \pm 1,51$ \\
\hline Hematocrit (\%) & $39-52$ & $27-49$ & $27-52$ & $44,67 \pm 3,03$ & $38,81 \pm 3,81$ & $42,14 \pm 4,46$ \\
\hline Committee exam score & $41-94$ & $38-92$ & $38-94$ & $67,23 \pm 12,66$ & $72,83 \pm 10,93$ & $69,64 \pm 12,22$ \\
\hline $\begin{array}{c}\text { Dairy products } \\
\text { consumption score }\end{array}$ & $20-626$ & $66-544$ & $20-626$ & $171,87 \pm 112,81$ & $200,78 \pm 120,51$ & $183,63 \pm 116,22$ \\
\hline $\begin{array}{l}\text { Meat products consumption } \\
\text { score }\end{array}$ & $32-618$ & $69-326$ & $32-618$ & $198,09 \pm 104,85$ & $174,59 \pm 65,97$ & $188,54 \pm 91,37$ \\
\hline $\begin{array}{l}\text { Vegetable consumption } \\
\text { score }\end{array}$ & $4-630$ & $5-324$ & $4-630$ & $122,46 \pm 95,19$ & $142 \pm 70,89$ & $130,41 \pm 86,25$ \\
\hline Fruit consumption score & $4-544$ & $14-1160$ & $4-1160$ & $119,83 \pm 102,79$ & $177,19 \pm 189,45$ & $143,15 \pm 146,22$ \\
\hline Grain consumption score & $125-939$ & $119-643$ & $119-939$ & $382,83 \pm 173,46$ & $347,73 \pm 148,39$ & $368,56 \pm 163,79$ \\
\hline Fat consumption score & $4-586$ & $4-452$ & $4-586$ & $198,8 \pm 146,21$ & $171,54 \pm 109,63$ & $187,71 \pm 132,58$ \\
\hline Sugar consumption score & $1-362$ & $7-270$ & $1-362$ & $127,43 \pm 81,08$ & $100,16 \pm 62,46$ & $116,34 \pm 74,92$ \\
\hline
\end{tabular}

(BMI: Body mass index, MET: Metabolic equivalent)

There was no significant difference between the weekly MET $/ \mathrm{min}$ values of males and females $(\mathrm{p}=0.059)$. In addition, no significant correlation was found between weekly $\mathrm{MET} / \mathrm{min}$ values and academic success $(p=0.536)$ (Table 4). 
The categorical results of physical activity levels are provided in Tables 2 and 3.

Table II: Statistical results for categorized variables $\left({ }^{*} p<0.05\right)$

\begin{tabular}{|c|c|c|c|c|c|c|}
\hline Characteristics & Category & $(\%)$ & $\mathbf{n}$ & Grade average \pm SD & $\begin{array}{c}t \text { value ( } \mathrm{t} \text { test) } \\
\text { / F value (ANOVA) }\end{array}$ & p value \\
\hline \multirow{2}{*}{ Gender } & male & 57 & 69 & $67,23 \pm 12,660$ & \multirow{2}{*}{$-2,550$} & \multirow{2}{*}{$0,012^{*}$} \\
\hline & female & 43 & 52 & $72,83 \pm 10,931$ & & \\
\hline \multirow{3}{*}{ Physical activity } & low & 20 & 18 & $66,11 \pm 11,842$ & \multirow{3}{*}{1,542} & \multirow{3}{*}{0,22} \\
\hline & moderate & 63 & 56 & $71,71 \pm 11,646$ & & \\
\hline & high & 17 & 15 & $68,93 \pm 14,099$ & & \\
\hline \multirow{2}{*}{ BMI $\left(\mathrm{kg} / \mathrm{m}^{2}\right)$} & $25>$ & 74 & 60 & $70,08 \pm 12,407$ & \multirow{2}{*}{0,725} & \multirow{2}{*}{0,471} \\
\hline & $25 \leq$ & 26 & 21 & $67,81 \pm 12,291$ & & \\
\hline \multirow{2}{*}{ Body fat ratio (\%) } & normal & 21 & 17 & $65,00 \pm 14,722$ & \multirow{2}{*}{$-1,709$} & \multirow{2}{*}{0,091} \\
\hline & high & 79 & 64 & $70,69 \pm 11,465$ & & \\
\hline \multirow{2}{*}{ Smoking } & yes & 29 & 26 & $64,88 \pm 11,504$ & \multirow{2}{*}{$-2,756$} & \multirow{2}{*}{$0,007^{*}$} \\
\hline & no & 71 & 65 & $72,49 \pm 12,047$ & & \\
\hline \multirow{2}{*}{ Alcohol } & yes & 40 & 36 & $67,83 \pm 12,225$ & \multirow{2}{*}{$-1,569$} & \multirow{2}{*}{0,12} \\
\hline & no & 60 & 55 & $71,95 \pm 12,227$ & & \\
\hline \multirow{2}{*}{ Vitamin } & yes & 21 & 19 & $68,84 \pm 13,035$ & \multirow{2}{*}{$-0,585$} & \multirow{2}{*}{0,56} \\
\hline & no & 79 & 72 & $70,71 \pm 12,196$ & & \\
\hline \multirow{2}{*}{ Main meal } & 1 or 2 & 35 & 32 & $67,66 \pm 10,703$ & \multirow{2}{*}{$-1,529$} & \multirow{2}{*}{0,13} \\
\hline & 3 & 65 & 59 & $71,76 \pm 12,979$ & & \\
\hline \multirow{2}{*}{ Adding sugar to drink } & no & 66 & 60 & $72,43 \pm 11,040$ & \multirow{2}{*}{2,332} & $0022 *$ \\
\hline & yes & 34 & 31 & $66,23 \pm 13,776$ & & $0,0<2^{2}$ \\
\hline & $<1500 \mathrm{ml}$ & 47 & 43 & $67,95 \pm 12,660$ & -1.752 & \\
\hline Dally water & $\geq 1500 \mathrm{ml}$ & 53 & 48 & $72,44 \pm 11,745$ & $-1,752$ & 0,083 \\
\hline & none & 11 & 10 & $76,5 \pm 10,648$ & & \\
\hline Daily tea & $1-2$ cup & 47 & 43 & $70,63 \pm 12,507$ & 1,791 & 0,173 \\
\hline & 3 or more cup & 42 & 38 & $68,34 \pm 12,244$ & & \\
\hline Daily coffee & no & 35 & 32 & $71,78 \pm 12,007$ & 0832 & 0407 \\
\hline Dally corree & yes & 65 & 59 & $69,53 \pm 12,524$ & 0,832 & $0,40 /$ \\
\hline Daily heyerage & no & 81 & 74 & $71,12 \pm 12,711$ & 1302 & 0196 \\
\hline Dany Deverage & yes & 19 & 17 & $66,82 \pm 10,070$ & 1,302 & 0,190 \\
\hline Dairy products & enough & 19 & 17 & $72,94 \pm 13,222$ & 0,973 & 0,333 \\
\hline Dany prouucts & not enough & 81 & 74 & $69,72 \pm 12,124$ & 0,97 & 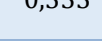 \\
\hline & enough & 71 & 65 & $70,91 \pm 12,219$ & 0795 & 0474 \\
\hline Meat products & not enough & 29 & 26 & $68,85 \pm 12,708$ & 0,195 & $0,4 / 4$ \\
\hline Vegetable & enough & 6 & 5 & $74,80 \pm 10,378$ & 0.843 & 0.401 \\
\hline vegetadie & not enough & 94 & 85 & $69,99 \pm 12,488$ & 0,843 & 0,401 \\
\hline Fruit & enough & 26 & 24 & $70,71 \pm 10,255$ & 0.193 & 0.858 \\
\hline & not enough & 74 & 67 & $70,18 \pm 13,055$ & & 0,050 \\
\hline & enough & 36 & 33 & $71,64 \pm 13,007$ & 0768 & 0445 \\
\hline Grain & not enough & 64 & 58 & $69,57 \pm 11,971$ & 0,168 & 0,445 \\
\hline
\end{tabular}

(BMI: Body mass index) 
Table III: Gender-separated results for categorized variables $\left({ }^{*} \mathrm{p}<0.05\right)$

\begin{tabular}{|c|c|c|c|c|c|c|c|c|c|c|c|}
\hline \multirow[b]{2}{*}{ Characteristics } & \multirow[b]{2}{*}{ Category } & \multicolumn{5}{|c|}{ Male } & \multicolumn{5}{|c|}{ Female } \\
\hline & & (\%) & $\mathbf{n}$ & $\begin{array}{c}\text { grade } \\
\text { average } \pm S D\end{array}$ & $\begin{array}{c}\text { t value } \\
\text { (t test) } \\
\text { / F } \\
\text { value } \\
\text { (ANOVA } \\
\text { ) }\end{array}$ & p value & (\%) & $\mathbf{n}$ & grade average \pm SD & $\begin{array}{l}t \text { value } \\
\text { ( } t \text { test) } \\
\text { / F } \\
\text { value } \\
\text { (ANOV } \\
\text { A) }\end{array}$ & p value \\
\hline \multirow{3}{*}{ Physical activity } & low & 25 & 13 & $61,92 \pm 9,535$ & \multirow{3}{*}{3,454} & \multirow{3}{*}{$0,04^{*}$} & 14 & 5 & $77,00 \pm 10,863$ & \multirow{3}{*}{1,256} & \multirow{3}{*}{0,298} \\
\hline & moderate & 52 & 27 & $72,22 \pm 11,882$ & & & 78 & 29 & $71,24 \pm 11,612$ & & \\
\hline & high & 23 & 12 & $66,17 \pm 14,528$ & & & 8 & 3 & $80,00 \pm 1,000$ & & \\
\hline \multirow{2}{*}{ BMI $\left(\mathrm{kg} / \mathrm{m}^{2}\right)$} & $25>$ & 70 & 33 & $67,88 \pm 14,333$ & \multirow{2}{*}{0,743} & \multirow{2}{*}{0,461} & 79 & 27 & $72,78 \pm 9,099$ & \multirow[b]{2}{*}{0,066} & \multirow[b]{2}{*}{0,949} \\
\hline & $25 \leq$ & 30 & 14 & $65,57 \pm 6,914$ & & & 21 & 7 & $72,29 \pm 19,102$ & & \\
\hline \multirow{2}{*}{ Body fat ratio } & normal & 23 & 11 & $59,55 \pm 13,909$ & \multirow{2}{*}{$-2,428$} & \multirow{2}{*}{$0,019^{*}$} & 18 & 6 & $75,00 \pm 11,009$ & \multirow{2}{*}{0,541} & \multirow[b]{2}{*}{0,592} \\
\hline & high & 77 & 36 & $69,53 \pm 11,305$ & & & 82 & 28 & $72,18 \pm 11,703$ & & \\
\hline \multirow{2}{*}{ Smoking } & yes & 31 & 17 & $62,35 \pm 12,604$ & \multirow{2}{*}{$-2,575$} & \multirow{2}{*}{$0,013^{*}$} & 24 & 9 & $69,67 \pm 7,517$ & \multirow[b]{2}{*}{$-0,937$} & \multirow[b]{2}{*}{0,355} \\
\hline & no & 69 & 37 & $71,57 \pm 12,036$ & & & 76 & 28 & $73,71 \pm 12,171$ & & \\
\hline & yes & 41 & 22 & $65,77 \pm 11,856$ & 1204 & 172 & 38 & 14 & $71,07 \pm 12,524$ & 0604 & \\
\hline RICU1101 & no & 59 & 32 & $70,66 \pm 13,300$ & $-1,304$ & $0,1 / 2$ & 62 & 23 & $73,74 \pm 10,580$ & $-0,094$ & 0,492 \\
\hline Vitamin & yes & 19 & 10 & $68,70 \pm 13,905$ & ( & 0092 & 24 & 9 & $69,00 \pm 12,835$ & 1147 & \\
\hline vilatiniti & no & 81 & 44 & $68,66 \pm 12,764$ & 0,00 & ניחנים & 76 & 28 & $73,93 \pm 10,680$ & $-1,1+1$ & 0,259 \\
\hline Moin morl & 1 or 2 & 31 & 17 & $64,47 \pm 11,641$ & 1652 & 0104 & 41 & 15 & $71,27 \pm 8,514$ & 0647 & \\
\hline Hiantincal & 3 or more & 69 & 37 & $70,59 \pm 13,061$ & $-1,030$ & 0,107 & 59 & 22 & $73,73 \pm 12,900$ & $-0,07 r$ & 0,522 \\
\hline Adding curor to drint & no & 57 & 31 & $71,16 \pm 12,396$ & 1685 & 0090 & 78 & 29 & $73,79 \pm 9,405$ & 1097 & \\
\hline תuй & yes & 43 & 23 & $65,30 \pm 12,938$ & 1,000 & 0,0 & 22 & 8 & $68,88 \pm 16,626$ & 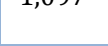 & 0,28 \\
\hline Dailunater & $<1500 \mathrm{ml}$ & 46 & 25 & $65,80 \pm 11,916$ & 1542 & 0120 & 49 & 18 & $70,94 \pm 13,388$ & 0.037 & \\
\hline 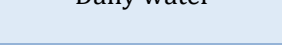 & $\geq 1500 \mathrm{ml}$ & 54 & 29 & $71,14 \pm 13,303$ & $-1,0+2$ & 0,123 & 51 & 19 & $74,42 \pm 8,834$ & $-0,931$ & 0,355 \\
\hline & None & 11 & 6 & $77,33 \pm 9,730$ & & & 11 & 4 & $75,25 \pm 13,376$ & & \\
\hline Daily tea & $1-2$ cup & 33 & 18 & $69,00 \pm 14,125$ & 1,760 & 0,182 & 67 & 25 & $71,80 \pm 11,358$ & 0,260 & \\
\hline & 3 or more & 56 & 30 & $66,73 \pm 12,199$ & & & 22 & 8 & $74,38 \pm 11,096$ & & 0,773 \\
\hline Doilus coffo & no & 48 & 26 & $73,00 \pm 12,576$ & 2505 & $0015 *$ & 16 & 6 & $66,50 \pm 7,868$ & 1507 & \\
\hline Dany cuire & yes & 52 & 28 & $64,64 \pm 11,942$ & 2,505 & 0,010 & 84 & 31 & $73,94 \pm 11,512$ & $-1,501$ & 0,141 \\
\hline Daily beyorage & no & 80 & 43 & $69,40 \pm 13,322$ & 0822 & 0415 & 84 & 31 & $73,52 \pm 11,596$ & 0965 & \\
\hline Dany Deverage & yes & 20 & 11 & $65,82 \pm 10,861$ & $0,0<2$ & 0,710 & 16 & 6 & $68,67 \pm 9,070$ & 0,700 & 0,341 \\
\hline Dairy productc & enough & 17 & 9 & $72,33 \pm 15,851$ & 0.937 & 0353 & 22 & 8 & $73,63 \pm 10,555$ & 0251 & \\
\hline Dairy pivucies & not enough & 83 & 45 & $67,93 \pm 12,239$ & 0,93 & 0,353 & 78 & 29 & $72,48 \pm 11,612$ & 0,251 & 0,803 \\
\hline Mont produrts & enough & 74 & 40 & $69,80 \pm 13,812$ & 1000 & 0277 & 68 & 25 & $72,68 \pm 9,100$ & 0030 & \\
\hline iviedt piouncts & not enough & 26 & 14 & $65,43 \pm 9,230$ & 1,090 & 0,271 & 32 & 12 & $72,83 \pm 15,302$ & $-0,030$ & 0,97 \\
\hline Womato & enough & 2 & 1 & 71,00 & Not $m$ l & lotod & 11 & 4 & $75,75 \pm 11,730$ & $0-72$ & \\
\hline vegetadie & not enough & 98 & 53 & $68,62 \pm 12,965$ & INot cal te the & ulated & 89 & 32 & $72,25 \pm 11,495$ & $0,5 / 3$ & 0,57 \\
\hline Fruit & enough & 17 & 9 & $66,22 \pm 10,533$ & 0622 & 0527 & 41 & 15 & $73,40 \pm 9,417$ & 0205 & \\
\hline rivit & not enough & 83 & 45 & $69,16 \pm 13,309$ & $-0,0<2$ & 0,537 & 59 & 22 & $72,27 \pm 12,555$ & 0,295 & 0,77 \\
\hline Crain & enough & 26 & 14 & $66,14 \pm 14,852$ & 0952 & 0290 & 51 & 19 & $75,68 \pm 10,034$ & 1681 & \\
\hline Gidm & not enough & 74 & 40 & $69,55 \pm 12,151$ & $-0,032$ & 0,390 & 49 & 18 & $69,61 \pm 11,907$ & 1,001 & 0,102 \\
\hline
\end{tabular}

(BMI: Body mass index) 
The committee exam scores of males with moderate physical activity levels were found to be significantly higher than males with low physical activity levels $(\mathrm{p}=0.043)$ (Figure 1).

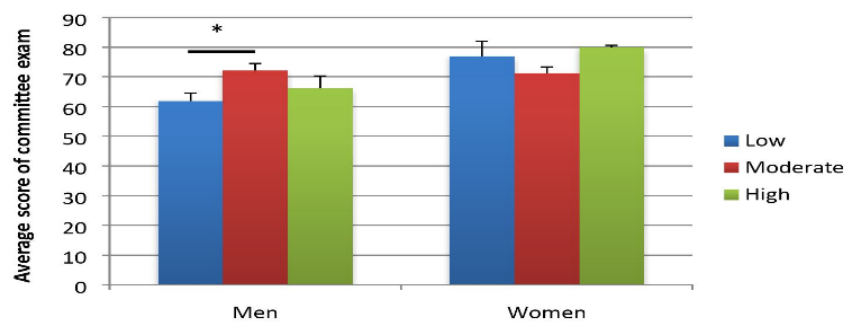

Figure 1: The effects of physical activity levels on academic achievement. * $\mathrm{p}<0.05$

A moderate positive correlation was determined between sedentary behavior and exam scores ( $p=0.012 \mathrm{r}=0.265)$ (Figure 2).

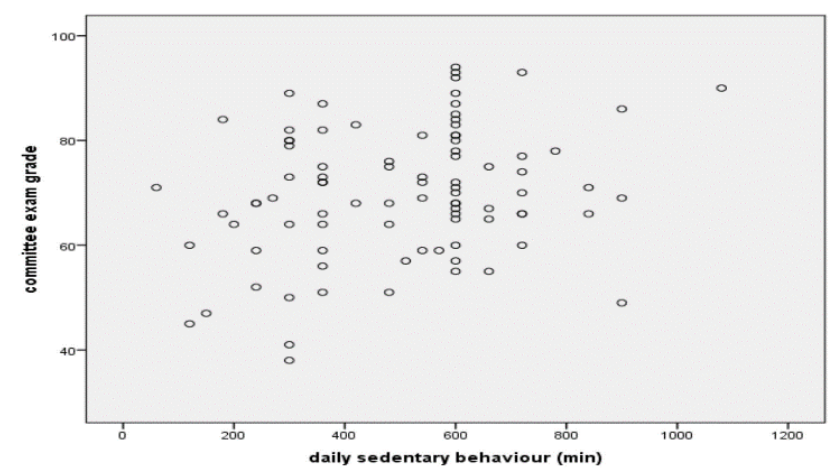

Figure 2: Correlation between sedentary behavior and academic achievement.

\section{Body Composition Results}

The results of students' height, weight, body mass index, muscle and fat ratios are provided in Table 1. The categorized results of BMI and body fat ratio are shown in Tables 2 and 3.

There was no statistically significant difference between the exam scores of the students with $\mathrm{BMI}<25$ and the students with $\mathrm{BMI} \geq 25$ (Table2 , Figure 3). No significant difference was detected in the exam scores of the student groups with normal and high fat ratio $(\mathrm{p}=0.091)$ (Figure-3).

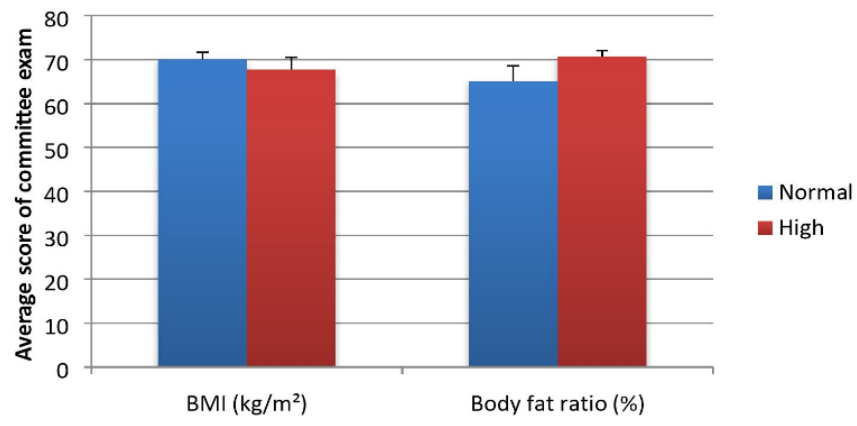

Figure 3: The effects of BMI $(\mathrm{kg} / \mathrm{m} 2)$ and body fat ratio (\%) on academic achievement. BMI: Body mass index.

The exam scores were not correlated with BMI and body fat ratios (Table 4).

Table IV: Results of correlation analyses between continuous variables and academic achievement $(* \mathrm{p}<0.05)$

\begin{tabular}{|c|c|c|c|c|c|c|}
\hline \multirow{2}{*}{ Parameter } & \multicolumn{2}{|c|}{ Male } & \multicolumn{2}{c|}{ Female } & \multicolumn{2}{c|}{ Total } \\
\cline { 2 - 6 } & Correlation coefficient $(r)$ & $p$ value & Correlation coefficient $(r)$ & $p$ & Correlation coefficient $(r)$ \\
value value
\end{tabular}




\section{Results of Eating Habits}

The data on students' smoking, alcohol usage, vitamin, water, tea, coffee, beverage and main meal consumptions, as well as adding sugar to their drinks are presented in Tables 2 and 3.

Non-smokers were more successful than smokers $(\mathrm{p}=0.007)$. When compared to those adding sugar to their drinks, the ones who did not add sugar to their drinks $(\mathrm{p}=0.022)$ were observed to be more successful. Additionally, male students who did not consume coffee were also found to be more successful than those consuming coffee $(\mathrm{p}=0.015)$ (Figure 4$)$.

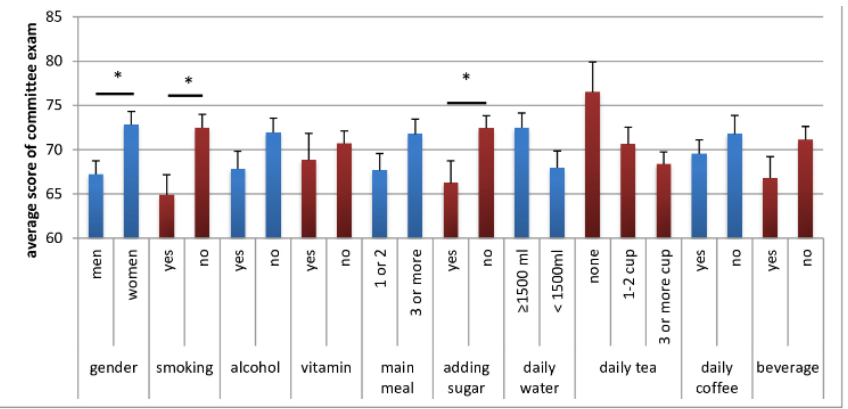

Figure 4: The effects of eating habits on academic achievement. * $\mathrm{p}<0.05$

\section{Results of food consumption frequency}

There was no significant difference between the exam scores of sufficient and insufficient consumers (Figure 5). Additionally, no significant correlation was observed in the consumption scores of food groups and exam scores (Table 4).

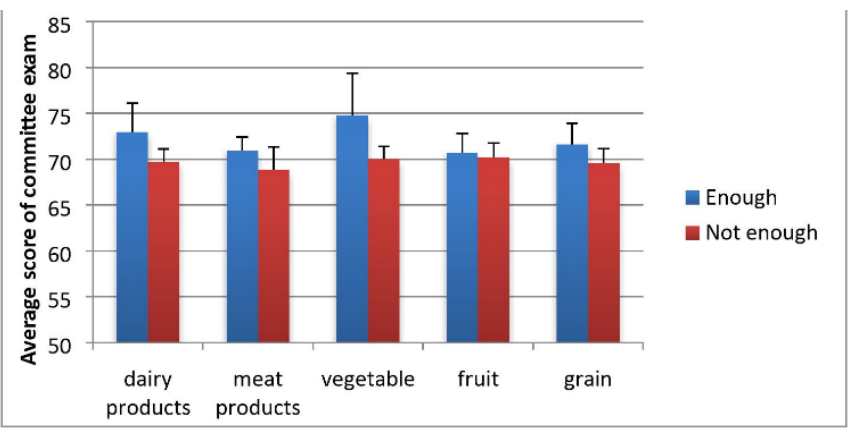

Figure 5: The effects of food consumption frequencies on academic achievement.

\section{DísCUSSín}

In this study, we evaluated hemoglobin levels, body compositions, nutritional habits, food consumption frequencies and physical activity levels among medical students, and investigated the effects of these factors on academic achievement. Our findings indicate that female students, non-smokers, those who do not consume sugary-beverages, male students with moderate physical activity levels coffeenonconsuming males were found to be more successful than their counterparts. Interestingly, sedentary behavior was associated with an increase in exam scores. On the other hand, hemoglobin levels, food consumption frequencies and body composition did not affect the academic achievement of medical students.

Although smoking rates are decreasing in Turkey as well as in many other countries, they are known to still be high among medical students $^{8}$. In addition to the well-known deleterious effects of smoking on public health, it also adversely affects cognitive functions. Moreover, these effects have also been demonstrated in young adults 9 . In this study, we observed that students who smoked were not as successful as non-smokers. Although there is not enough data on the effects of cigarette smoking on academic achievement in university students in the literature, our result is not surprising as smoking has a negative impact on cognitive functions.

Our results demonstrated that female students were more successful than male students; gender is known to be one of the factors affecting academic success in medical schools. In the literature, despite several varying results in studies examining the effect of gender on success in medical education, it is reported that female students generally perform better ${ }^{10}$. The reasons for these differences in academic acheivement is thought to be due to the 
differences both in the education systems applied in medical schools and in the learning styles of males and females. In addition, a study stated that since women tend to be more anxious in terms of doability, they study harder and more meticulously to deal with this uncertainty. This helps to explain the success of female students in medical education ${ }^{11}$.

In our study, we observed that the consumption of sugary beverages negatively affected academic achievement in medical students. Medical students usually restrict both their social lives and sleep times in order to allocate suffient study time. Thus, they tend to consume caffeinated beverages such as tea and coffee. For students who have the habit of adding sugar to their drinks, this results in an unconsciously excessive intake of simple sugar. Sugary beverage consumption yields negative effects on cognitive functions by affecting various inflammatory mechanisms and neurotrophic factors in the brain; this has been demonstrated in both experimental studies and among various age groups ${ }^{12,13}$.

Physical activity has a positive effect on cognitive functions and academic success by increasing brain perfusion, glucose and lipid metabolism, neurotrophic factor levels and gray matter volume of the hippocampus and frontal cortex $^{14}$. However, there are studies in the literature stating that the improvement in cognitive skills in response to increasing exercise levels does not indicate linearity and draws an inverted-U like curve ${ }^{15,16}$. In our study, male students with moderate physical activity levels were found to be more successful than males with low physical activity levels. Our results support the tenet that similar to cognitive functions, physical activity also has a dose-dependent effect on academic achievement.

It is known that caffeine increases alertness and cognitive performance. Although caffeine reduces headaches with its vasoconstrictor effect, in chronic use, it has been shown to trigger headaches and cause psychiatric symptoms such as anxiety and depression; this in turn negatively affects academic success ${ }^{17,18}$. In our study, male students who consumed coffee every day were found to be more unsuccessful than those who did not. The gender-dependent differences in consumption rates or total caffeine intake doses and duration of other various caffeinated products such as tea, chocolate, and energy drinks may explain the different results we observed according to gender.

In this study, we found that sedentary behavior increased the academic achievement of medical students. Sedentary behavior refers to the situation of sitting or lying down while awake in which energy expenditure is very low. Many activities such as reading, studying, resting, watching TV, and computer use are included in sedentary behavior. A study reported that a long duration of screen viewing negatively affected cognitive skills and academic success ${ }^{19}$. However, due to the academic load, medical students are likely to study for most of the duration of time they spend in a sedentary manner. It is known that spending a long time studying and reading is associated with an increase in academic achievement ${ }^{20}$.

Hemoglobin, which is associated with cognitive skills in terms of its content and function, has been reported to cause a 1.73 point decrease in IQ scores in case of $1 \mathrm{gr} / \mathrm{dl}$ decrease in its levels ${ }^{21}$. Thus, hemoglobin is a fundamental factor that can influence cognitive functions via IQ scores. However, our study population was students older than 18 who were medical students with a higer level of intelligence. In addition, low hemoglobin levels can cause stress, headaches, fatigue and hypoxia, and can consequently adversely affect physical endurance, immune responses, temperature regulation and energy metabolism. This in turn can reduce cognitive performance ${ }^{22,23}$. In the 
literature, various studies usually comparing anemic and non-anemic student groups have reported hemoglobin level has a positive or null effect on academic achievement ${ }^{24}$. In our study, the hemoglobin levels of the vast majority of the students were within the normal range and we did not detect a relationship between academic achievement and hemoglobin levels. This result may be due to our focusing on an isolated student group such as medical students, or due to the fact that the impact of fluctuations observed in the normal hemoglobin concentrations on academic success are negligible.

Dietary nutrients can affect cognitive functions through many different mechanisms. The consumption of whole grains, dairy products and foods with high antioxidant content such as vegetables and fruits have been shown to yield beneficial effects on cognition and academic achievement ${ }^{25,26}$. In contrast, the "Western diet" rich in saturated fat and refined carbohydrates, has a negative effect on learning-memory ${ }^{27}$. In our study, there was no correlation between the consumption scores of the food groups and academic achievement in medical students. As a consequence of moving to other cities to study at university, students usually start to live apart from their families and their ongoing eating habits change dramatically. Therefore, it may be possible to observe cognitive changes depending on the consumption of food groups by planning more time-extended studies.

Today, the consumption of calorie-rich and nutrient-poor foods is rapidly increasing among children and youth. Thus, individuals who are uncertain about meeting their micronutrient needs by diet tend to use multivitamin-mineral supplements. A study reported that only the students whose diets had a low micro-nutrient content benefited from the micronutrient supplements ${ }^{28}$. Another study indicated these supplements were found to be ineffective on school performance ${ }^{29}$. Consistently, in our study, the use of vitamin supplements did not affect academic achievement. However, our study has some limitations in that the participants were not questioned if there was a micronutrient deficiency and neither were the contents of the vitamin preparations mentioned.

In our study, we did not detect a relationship between BMI and academic achievement in medical students. However, BMI is a limited method on its own. The determination of body fat ratio in addition to the calculation of BMI yields more reliable results. In the present study, no correlation was found between body fat and academic achievement, whereas male students with a high body fat ratio were more successful than the males in the normal range. In Turkey, the vast majority of students leave their homes to attend university and usually stay in student dormitories during the first years. Thus, they expereince difficulty in consuming sufficient and balanced nutrition. In this study, we observed that compared to female students, males insufficiently consume all food groups except meat products. This result supports the possibility of males being more affected by malnutrition during the university life adaptation phase. The possible explanation of our results may be that compared to malnutrition, excessive energy intake results in better consequences in terms of cognitive skills such as perception, learning and study performance. In accordance with our results, another study reported the increase in adiposity was associated with good nutritional status which was emphasized to be the main determinant of cognitive functions especially in low income countries ${ }^{30}$. In our study, according to the WHO classification of BMI, students with a high fat ratio belonged to the overweight category with the exceptance of 2 males who were considered obese. Since the participants' fat ratio distribution was within a restricted range, we were unable to find a correlation 
between body fat ratio and academic achievement in medical students.

\section{CONCLUSION}

In summary, while being male, smoking, coffee consumption and adding sugar to their drinks were detected to be some factors decreasing academic achievement, a moderate level of physical activity enhanced academic performance in medical students. However, the consumption frequencies of food groups, hemoglobin levels and BMI have been found to be ineffective on academic success. In addition, a possible explanation of the positive relationship between sedentary behavior and academic achievement might be due to the fact the time spent in a sedentary manner often corresponds to the time medical students allocate to studying. It should also be emphasized that these factors affect academic success of medical students in a genderdependent manner. In this study, we generally evaluated "changeable" factors with the potential to affect academic achievement and based on these, we aimed to provide some recommendations to medical students. It is clear that, in additon to these factors, psychological, social and environmental factors also play important roles on academic success. Although a large number of factors were addressed in this study, further studies equipped with biopsychosocial factors are needed to elucidate all the determinants of academic achievement during medical school.

Our study has some limitations. Firstly, academic achievement was evaluated based on a single exam and this may not fully reflect overall success. Secondly, this study was implemented only among medical school students. Therefore, the results of this study cannot be generalized to encompass all undergraduate students. Thirdly, as it has a cross-sectional design, this study cannot reveal the cause-effect relationship. Fourthly, since the data on nutritional status and physical activity was collected by the survey method, bias probability cannot be excluded. The strength of this study is that it was implemented on a homogeneous student population including both genders.

Acknowledgements: We would like to thank Prof Dr Mesut Saçkes and Dr Soner Güneş for their substantial support in the statistical evaluation of this study. We also gratefully thank two of our medical students, Ibrahim Demirhan and Tarık Özkan for their valuable contributions in the data collection phase.

Ethics Committee Approval: The study protocol was approved by the Clinical Research Ethics Committee of Balıkesir University Faculty of Medicine (Decision date: 30.01.2019, Number: 2019/24).

Conflict of Interest: The authors declared no conflicts of interest.

Financial Disclosure: The authors declared that this study has received no financial support.

\section{REFERENCES}

1. Dubuc MM, Aubertin-Leheudre M, Karelis AD. Relationship between Academic Performance with Physical, Psychosocial, Lifestyle, and Sociodemographic Factors in Female Undergraduate Students. Int J Prev Med. 2017; 8: 22.

2. World Health Organisation (WHO). Body Mass Index-BMI. https://bit.ly/32xqnDt

3. Lee R, Nieman D: Anthropometry: Nutritional Assessment, vol. 119. USA: Brown and Benchmark; 1993.

4. Pekcan GŞ, N.; Baş, M.: Türkiye Beslenme Rehberi TÜBER 2015. Ankara: T.C. Sağlık Bakanlığı; 2016.

5. Rakıcıoğlu N, Samur G, Başoğlu S: Diyetisyenler İçin Hasta İzlem Rehberi/Ağırlık Yönetimi El Kitabı. Ankara: T.C. Sağlık Bakanlığı Halk Sağlığı Genel Müdürlüğü; 2017.

6. Saglam M, Arikan H, Savci S et al. International physical activity questionnaire: reliability and 
validity of the Turkish version. Percept Mot Skills. 2010; 111: 278-84.

7. WHO: Haemoglobin Concentrations for the Diagnosis of Anaemia and Assessment of Severity Geneva: World Health Organization; 2011.

8. Baykan Z, Naçar M. Tıp fakültesi öğrencilerinin sigara kullanımı ve tütün kanununa ilişkin görüşleri. Dicle Tıp Dergisi. 2014; 41: 483-90.

9. Paul RH, Brickman AM, Cohen RA et al. Cognitive status of young and older cigarette smokers: data from the international brain database. J Clin Neurosci. 2006; 13: 457-65.

10. Ferguson E, James D, Madeley L. Factors associated with success in medical school: systematic review of the literature. BMJ (Clinical research ed). 2002; 324: 952-7.

11. Haidinger G, Frischenschlager 0 , Mitterauer L. Reliability of predictors of study success in medicine. Wien Med Wochenschr. 2006; 156: 41620.

12. Muñoz-García MI, Martínez-González MA, Martín-Moreno JM et al. Sugar-sweetened and artificially-sweetened beverages and changes in cognitive function in the SUN project. Nutritional Neuroscience. 2020; 23: 946-54.

13. Bleiweiss-Sande $\mathrm{R}$, Chui $\mathrm{K}$, Wright $\mathrm{C}$, et al. Associations between Food Group Intake, Cognition, and Academic Achievement in Elementary Schoolchildren. Nutrients. 2019; 11: 2722.

14. Naveed S, Lakka T, Haapala EA. An Overview on the Associations between Health Behaviors and Brain Health in Children and Adolescents with Special Reference to Diet Quality. International Journal of Environmental Research and Public Health. 2020; 17: 953.

15. Loprinzi PD, Edwards MK, Crush E, Ikuta T, Del Arco A. Dose-Response Association Between Physical Activity and Cognitive Function in a National Sample of Older Adults. American Journal of Health Promotion. 2018; 32: 554-60.

16. Bernard P, Doré I, Romain AJ, et al. Dose response association of objective physical activity with mental health in a representative national sample of adults: A cross-sectional study. PLoS One. 2018; 13.
17. Cappelletti S, Piacentino D, Sani G, Aromatario M. Caffeine: cognitive and physical performance enhancer or psychoactive drug? Curr Neuropharmacol. 2015; 13: 71-88.

18. Gilliland $\mathrm{K}$, Andress D. Ad lib caffeine consumption, symptoms of caffeinism, and academic performance. Am J Psychiatry. 1981; 138: 512-4.

19. Carson V, Hunter S, Kuzik N, et al. Systematic review of sedentary behaviour and health indicators in school-aged children and youth: an update. Appl Physiol Nutr Metab. 2016; 41: S240-65.

20. Haapala EA, Poikkeus AM, Kukkonen-Harjula K, et al. Associations of physical activity and sedentary behavior with academic skills--a follow-up study among primary school children. PLoS One. 2014; 9: e107031.

21. Stoltzfus RJ ML, Black RE: Iron deficiency anaemia. In: Comparative quantification of health risks: Global and regional burden of disease attributable to selected major risk factors. Volume 1, edn. Edited by Ezzati M LA, Rodgers A, Murray CJL; 2004: 163-209.

22. Shah RC, Wilson RS, Tang Y, et al. Relation of hemoglobin to level of cognitive function in older persons. Neuroepidemiology. 2009; 32: 40-6.

23. Schneider ALC, Jonassaint C, Sharrett AR et al. Hemoglobin, Anemia, and Cognitive Function: The Atherosclerosis Risk in Communities Study. The Journals of Gerontology: Series A. 2015; 71: 772-9.

24. Rani NA, Arasegowda R, Mukherjee P, Dhananjay SY. Prevalence of Nutritional Deficiency Anaemia and Its Impact on Scholastic Performance among Undergraduate Medical Students. J Clin Diagn Res. 2017; 11: Bc21-bc3.

25. Ptomey LT, Steger FL, Schubert MM, et al. Breakfast Intake and Composition Is Associated with Superior Academic Achievement in Elementary Schoolchildren. J Am Coll Nutr. 2016; 35: 326-33.

26. Ivanovic D, Vasquez M, Aguayo M, et al. Nutrition and education. III. Educational achievement and food habits of Chilean elementary and high school graduates. Arch Latinoam Nutr. 1992; 42: 9-14.

27. Jacka FN, Cherbuin N, Anstey KJ, Sachdev P, Butterworth P. Western diet is associated with a 
smaller hippocampus: a longitudinal investigation. BMC Med. 2015; 13: 215.

28. Benton D. Micro-nutrient supplementation and the intelligence of children. Neurosci Biobehav Rev. 2001; 25: 297-309.

29. Perlman AI, Worobey J, O'Sullivan Maillet J, et al. Multivitamin/Mineral supplementation does not affect standardized assessment of academic performance in elementary school children. J Am Diet Assoc. 2010; 110: 1089-93.

30. Veena SR, Hegde BG, Ramachandraiah S, et alK. Relationship between adiposity and cognitive performance in 9-10-year-old children in South India. Arch Dis Child. 2014; 99: 126-34. 